\title{
Comparison of Saliency-Based Sensorless Control Techniques for AC Machines
}

\author{
Fernando Briz, Member, IEEE, Michael W. Degner, Member, IEEE, Pablo García, Student Member, IEEE, and \\ Robert D. Lorenz, Fellow, IEEE
}

\begin{abstract}
This paper analyzes saliency-based sensorless control methods for ac machines. The paper focuses on three different methods, which utilize the following signals: negative-sequence carrier-signal current, zero-sequence carrier-signal voltage, and pulesewidth-modulation-switching zero-sequence voltage. Applicability of these methods for both rotor position estimation (tracking of rotor-position-dependent saliencies) and flux position estimation (tracking of flux-dependent saliencies) is studied for each method, as well as aspects of their implementation.
\end{abstract}

Index Terms-Flux angle estimation, rotor position estimation, saliency-based sensorless control.

\section{INTRODUCTION}

$\mathbf{S}$ ENSORLESS control techniques for ac machines that rely on the fundamental excitation have been shown to be capable of providing high-performance field-oriented control in the medium- to high-speed range [1]-[3]. Such techniques, however, fail in the low-speed range or for dc excitation. To overcome this limitation, sensorless control methods based on tracking the position of saliencies in the electric machines have been proposed. Such methods have the capability of providing accurate, high bandwidth, position, speed, and disturbance torque estimates and/or flux estimates over a wide speed range, including zero speed and frequency [4]-[18]. These techniques measure the response of the machine when a persistent, high-frequency excitation, distinct from the fundamental excitation, is applied via the inverter. The major differences between the methods are the type of high-frequency excitation and the signal processing used to estimate the rotor or flux position. The types of persistent excitation that have been proposed can be classified into two main categories.

Paper IPCSD-04-028, presented at the 2003 Industry Applications Society Annual Meeting, Salt Lake City, UT, October 12-16, and approved for publication in the IEEE TRANSACTIONS ON INDUSTRY APPLICATIONS by the Industrial Drives Committee of the IEEE Industry Applications Society. Manuscript submitted for review October 1, 2003 and released for publication April 6 , 2004. This work was supported in part by the Research, Technological Development and Innovation Programs of the Principado of Asturias-ERDF, under Grant PB-EXP01-24 and Grant PB02-055, and by the Spanish Ministry of Science and Technology-ERDF under Grant DPI2001-3815.

F. Briz and P. García are with the Department of Electrical, Computer and Systems Engineering, University of Oviedo, E-33204 Gijón, Spain (e-mail: fernando@isa.uniovi.es; pgarcia@isa.uniovi.es).

M. W. Degner is with Sustainable Mobility Technologies, Ford Motor Company, Dearborn, MI 48121-2053 USA (e-mail: mdegner@ford.com).

R. D. Lorenz is with the Departments of Mechanical Engineering and Electrical and Computer Engineering, University of Wisconsin, Madison, WI 53706 USA (e-mail: lorenz@engr.wisc.edu).

Digital Object Identifier 10.1109/TIA.2004.830768
1) One is the injection of a carrier signal (usually in the range of several hundred hertz) superimposed on the fundamental excitation and generally distinct from the pulsewidth-modulation (PWM) switching excitation created by the inverter [4]-[15]. This type of excitation usually takes one of two forms: 1) a constant amplitude voltage [4]-[9], [13]-[15] (current [10]) vector rotating in the stationary frame at the carrier frequency or 2) an amplitude-modulated, at the carrier frequency, voltage (current) vector(s) rotating in a frame synchronous to the estimated quantity [11], [12].

2) The other is excitation created by the PWM switching of the inverter, commonly using modified forms of PWM [3], [16] or applying specific switching states during the measurement period [17], [18].

With regard to the number and type of signals measured, techniques using two phase currents, three phase currents, phase voltages and/or the neutral voltage have been reported, with a variety of alternatives in the signal processing used to extract the desired information [4]-[18].

The primary goal of this paper is the analysis and comparison of saliency-based sensorless control methods for the estimation of rotor position and/or flux angle in ac machines. The paper begins by modeling saliencies, as well as the measurable effects that they produce, when a high-frequency excitation signal is applied to a machine. The paper then focuses on three different sensorless control methods: 1) negative-sequence carrier-signal-current-based methods [4]-[9]; 2) zero-sequence carrier-signal-voltage-based methods [13], [15]; and 3) PWMswitching zero-sequence-voltage-based methods [3], [16]. Differences in the generation, acquisition, and processing of the signal needed for estimating the desired rotor position or flux angle are also analyzed.

\section{CARRIER-SIGNAL-EXCITATION-BASED SENSORLESS METHODS}

Carrier-signal-based sensorless methods inject a high-frequency excitation (voltage [4]-[9], [11]-[15] or current [10]), whose interaction with the machine saliencies produces specific frequency components in measurable electrical variables (phase/zero-sequence currents [4]-[9], [11], [12], [14] and voltages [10], [13], [15]), which are used to estimate the position of the saliency. One of the most common forms of high-frequency carrier-signal excitation is the use of a single rotating vector [4]-[9] and is the basis for the analysis provided 
TABLE I

INDUCTION Motors PARAMETERS (All HAVE Four Poles)

\begin{tabular}{l|c|c|c|c}
\hline & MOTOR \#1 & MOTOR \#2 & MOTOR \#3 & MOTOR \#4 \\
\hline \# Stator slots & 36 & 36 & 36 & 24 \\
\hline \# Rotor slots & 28 & 26 & 28 & 30 \\
\hline $\begin{array}{c}\text { Rotor slots } \\
\text { (all skewed) }\end{array}$ & Semi-closed & Semi-closed & \#1: Semi- closed & \#1: Semi-closed \\
& & & \#2: Closed & \#2: Closed \\
& & & $1.1 \mathrm{~kW}$ & $0.9 \mathrm{~kW}$ \\
\hline Power rating & $1.1 \mathrm{~kW}$ & $0.75 \mathrm{~kW}$ & \#3: Engineered \\
\hline
\end{tabular}

in this paper on carrier-signal-excitation-based methods. Included in this analysis are methods that utilize the resulting negative-sequence carrier-signal current [4]-[9] and zero-sequence carrier-signal voltage [13], [15]. Although other forms of carrier-signal excitation, such as the amplitude-modulated methods [11], [12] and carrier-signal-current methods [10], are not included in the analysis provided in this paper, due to space restrictions, their similarity to the analyzed methods is expected to result in similar behavior and conclusions.

\section{A. Modeling and Behavior of Salient Machines}

At frequencies near the carrier signal the general model for the induction machine can be simplified to one consisting of only the machine inductances (1), where $L_{A B C}$ is a $3 \times 3$ matrix and can be modeled as consisting of four submatrices (2)

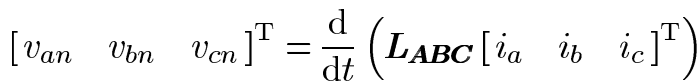

$$
\begin{aligned}
& L_{A B C}=L_{m \_c o n s t a n t}+L_{m \_v a r y i n g} \\
& +L_{l \_c o n s t a n t}+L_{l \_v a r y i n g} .
\end{aligned}
$$

$\boldsymbol{L}_{\boldsymbol{m} \_ \text {constant }}$ and $\boldsymbol{L}_{\boldsymbol{m} \_\boldsymbol{v} a \boldsymbol{r} y i n g}$ model the constant and varying portions of the phase mutual inductances (diagonal terms are zero).

$L_{l_{-} \text {constant }}$ and $L_{l_{-v a r y i n g}}$ model the constant and varying portions of the phase self-inductances, respectively (off-diagonal terms are equal to zero).

While the general solution for the behavior of the machine can be obtained from (1) and (2), some further simplifications are possible based on the values for these inductances in relation to the machine design and carrier-signal frequency. To asses the contribution of each term in (2) to the overall inductance, tests were conducted on motor \#1 in Table I. The following values for each entry in these inductance submatrices was determined for a carrier frequency of $\omega_{c}=500 \mathrm{~Hz}: L_{m}$ constant $=$ $0.57 \mathrm{mH}, L_{m \_ \text {varying }}=0.027 \mathrm{mH}, L_{\text {l_constant }}=28.9 \mathrm{mH}$, and $L_{l \_ \text {varying }}=1.18 \mathrm{mH}$.

From these values it can be seen that the mutual inductances between the phases are relatively small in comparison to the self-inductances. Based on this, the model shown in (2) can be simplified by only considering the self-inductance terms, which at the carrier frequency are approximately equal to the stator transient inductance. With this assumption, a further assumption that the varying inductances consist of a single harmonic component, and the fact that for the operating speeds of interest the carrier-signal frequency is significantly larger than the fundamental frequency (the time rate of change of the inductance in (1) can be ignored), the machine model can be simplified to (3)-(5) (see Fig. 2)

$$
\begin{aligned}
& v_{a n}=\left(\Sigma L_{\sigma s}+2 \Delta L_{\sigma s} \cos \left(h \theta_{e}\right)\right) \frac{\mathrm{d} i_{a}}{\mathrm{~d} t} \\
& v_{b n}=\left(\Sigma L_{\sigma s}+2 \Delta L_{\sigma s} \cos \left(h\left(\theta_{e}-\frac{2 \pi}{3}\right)\right)\right) \frac{\mathrm{d} i_{b}}{\mathrm{~d} t} \\
& v_{c n}=\left(\Sigma L_{\sigma s}+2 \Delta L_{\sigma s} \cos \left(h\left(\theta_{e}-\frac{4 \pi}{3}\right)\right)\right) \frac{\mathrm{d} i_{c}}{\mathrm{~d} t}
\end{aligned}
$$

where the following apply.

- $\Sigma L_{\sigma s}$ and $\Delta L_{\sigma s}$ are the average and differential stator transient inductances, being equal to $L_{l_{-c o n s t a n t}}$ and $L_{l_{\text {_varying }}} / 2$ in (2), respectively. The definition of the differential inductance $\Delta L_{\sigma s}$ in (3)-(5) (note the constant 2) was chosen as a matter of convenience to keep the results obtained when transforming the three-phase system to an equivalent two-phase system consistent with the nomenclature widely used in the literature [4], [5].

- $h$ is the harmonic order of the saliency relative to electrical angular units.

- $\theta_{e}$ is the angular position of the saliency in electrical radians.

The zero-sequence voltage (6) can be calculated directly using (3)-(5) and (7), with the result shown in (8). Note the inductances $L_{a}, L_{b}$, and $L_{c}$ in (8) stand for the inductance terms in (3)-(5), respectively

$$
\begin{aligned}
v_{0 s}^{s} & =\frac{1}{3}\left(v_{a n}+v_{b n}+v_{c n}\right) \\
\frac{\mathrm{d} i_{a}}{\mathrm{~d} t}+\frac{\mathrm{d} i_{b}}{\mathrm{~d} t}+\frac{\mathrm{d} i_{c}}{\mathrm{~d} t} & =0 \\
v_{0 s}^{s} & =-\frac{v_{a} L_{b} L_{c}+v_{b} L_{a} L_{c}+v_{c} L_{a} L_{b}}{L_{b} L_{c}+L_{a} L_{c}+L_{a} L_{b}} .
\end{aligned}
$$

By transforming (3)-(5) to an equivalent $q d 0$ model, (9) can be obtained

$$
\left[\begin{array}{lll}
v_{q s}^{s} & v_{d s}^{s} & v_{0 s}^{s}
\end{array}\right]^{\mathrm{T}}=L_{\sigma q d 0 s}^{s} \frac{\mathrm{d}}{\mathrm{d} t}\left[\begin{array}{lll}
i_{q s}^{s} & i_{d s}^{s} & i_{0 s}^{s}
\end{array}\right]^{\mathrm{T}} .
$$

While a general solution for the currents and the zero-sequence voltage can be obtained from (9), much simpler solutions are obtained by solving (9) for particular values of $h$ using the following equations for the inductances (10)-(12) [5]:

1) for $h=1,4,7, \ldots$

$$
\begin{aligned}
& L_{\sigma q d 0 s}^{s}=\Sigma L_{\sigma s}\left[\begin{array}{lll}
1 & 0 & 0 \\
0 & 1 & 0 \\
0 & 0 & 1
\end{array}\right] \\
& +\Delta L_{\sigma s}\left[\begin{array}{ccc}
\cos \left(h \theta_{e}\right) & \sin \left(h \theta_{e}\right) & 2 \cos \left(h \theta_{e}\right) \\
\sin \left(h \theta_{e}\right) & -\cos \left(h \theta_{e}\right) & -2 \sin \left(h \theta_{e}\right) \\
\cos \left(h \theta_{e}\right) & -\sin \left(h \theta_{e}\right) & 0
\end{array}\right]
\end{aligned}
$$

2) for $h=2,5,8, \ldots$

$$
\begin{aligned}
& L_{\sigma q d 0 s}^{s}=\Sigma L_{\sigma s}\left[\begin{array}{lll}
1 & 0 & 0 \\
0 & 1 & 0 \\
0 & 0 & 1
\end{array}\right] \\
& +\Delta L_{\sigma s}\left[\begin{array}{ccc}
\cos \left(h \theta_{e}\right) & -\sin \left(h \theta_{e}\right) & 2 \cos \left(h \theta_{e}\right) \\
-\sin \left(h \theta_{e}\right) & -\cos \left(h \theta_{e}\right) & 2 \sin \left(h \theta_{e}\right) \\
\cos \left(h \theta_{e}\right) & \sin \left(h \theta_{e}\right) & 0
\end{array}\right]
\end{aligned}
$$


3) for $h=3,6,9, \ldots$

$L_{\sigma q d 0 s}^{s}=\Sigma L_{\sigma s}\left[\begin{array}{ccc}1 & 0 & 0 \\ 0 & 1 & 0 \\ 0 & 0 & 1\end{array}\right]+2 \Delta L_{\sigma s} \cos \left(h \theta_{e}\right)\left[\begin{array}{lll}1 & 0 & 0 \\ 0 & 1 & 0 \\ 0 & 0 & 0\end{array}\right]$.

Using (8)-(12) and applying a balanced, three-phase carriersignal voltage, (13)-(15), to the machine, the resulting carriersignal current and zero-sequence carrier-signal voltage for particular values of $h$ can be calculated as (16)-(21) as follows:

$$
\begin{aligned}
& v_{a}=V_{c} \cos \left(\omega_{c} t\right) \\
& v_{b}=V_{c} \cos \left(\omega_{c} t-\frac{2 \pi}{3}\right) \\
& v_{c}=V_{c} \cos \left(\omega_{c} t-\frac{4 \pi}{3}\right)
\end{aligned}
$$

1) for $h=1,4,7, \ldots$

$$
\begin{aligned}
i_{q d s_{-} c}^{s} & =-\mathrm{j} I_{c p} \mathrm{e}^{\mathrm{j} \omega_{c} t}-\mathrm{j} I_{c n} \mathrm{e}^{\mathrm{j}\left(-h \theta_{e}-\omega_{c} t\right)} \\
v_{0 s c}^{s} & \left.=V_{0 c h} \cos \left(\omega_{c} t-h \theta_{e}\right)-V_{0 c 2 h} \cos \left(\omega_{c} t+2 h \theta_{e}\right)\right)
\end{aligned}
$$

2) for $h=2,5,8, \ldots$

$$
\begin{aligned}
i_{q d s_{-} c}^{s} & =-\mathrm{j} I_{c p} \mathrm{e}^{\mathrm{j} \omega_{c} t}-\mathrm{j} I_{c n} \mathrm{e}^{\mathrm{j}\left(h \theta_{e}-\omega_{c} t\right)} \\
v_{0 s c}^{s} & \left.=V_{0 c h} \cos \left(\omega_{c} t+h \theta_{e}\right)-V_{0 c 2 h} \cos \left(\omega_{c} t-2 h \theta_{e}\right)\right)
\end{aligned}
$$

3) for $h=3,6,9, \ldots$

$$
\begin{aligned}
i_{q d s_{-} c}^{s} & =-\mathrm{j} \frac{V_{c}}{\omega_{c}} \frac{1}{\Sigma L_{\sigma s}+2 \Delta L_{\sigma s} \cos \left(h \theta_{e}\right)} \mathrm{e}^{\mathrm{j} \omega_{c} t} \\
v_{0 s c}^{s} & =0
\end{aligned}
$$

where $I_{c p}=\left(V_{c} / \omega_{c}\right)\left(\Sigma L_{\sigma s} /\left(\Sigma L_{\sigma s^{2}}-\Delta L_{\sigma s^{2}}\right)\right)$ is the magnitude of the positive-sequence carrier-signal current, $I_{c n}=\left(V_{c} / \omega_{c}\right)\left(\Delta L_{\sigma s} /\left(\Sigma L_{\sigma s^{2}}-\Delta L_{\sigma s^{2}}\right)\right)$ is the magnitude of the negative-sequence carrier-signal current, $V_{0 c h}=V_{c} \Sigma L_{\sigma s} \Delta L_{\sigma s} /\left(\Sigma L_{\sigma s^{2}}-\Delta L_{\sigma s^{2}}\right)$ is the magnitude of the $h \theta_{e}$ component of the zero-sequence carrier-signal voltage, and $V_{0 c 2 h}=$ $V_{c} \Delta L_{\sigma s^{2}} /\left(\Sigma L_{\sigma s^{2}}-\Delta L_{\sigma s^{2}}\right)$ is the magnitude of the $-2 h \theta_{e}$ component of the zero-sequence carrier-signal voltage.

From (20) and (21) it can be seen that for the case of $h=3,6, \ldots$ no zero-sequence carrier-signal voltage exists and the carrier-signal current is an amplitude-modulated positive-sequence component. It is also noted that, although (17) and (19) predict the presence of a secondary component of the zero-sequence carrier voltage, its magnitude $V_{0 c 2 h}$ is negligible when compared to $V_{0 c h}$ for $\Delta L_{\sigma s} \ll \Sigma L_{\sigma s}$, which is typically the case.

\section{B. Experimental Setup}

Fig. 1 shows the experimental setup used for the experiments presented in this paper. It consists of a current-regulated induction machine, with the carrier-signal voltage superimposed on the fundamental voltage. The rotor speed and, consequently, the slip and the load level of the machine, was set using a load dynamometer. A four-channel digital oscilloscope with extended

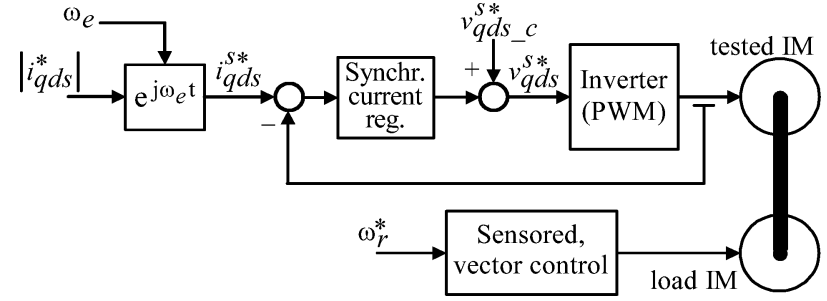

Fig. 1. Schematic representation of the experimental setup.

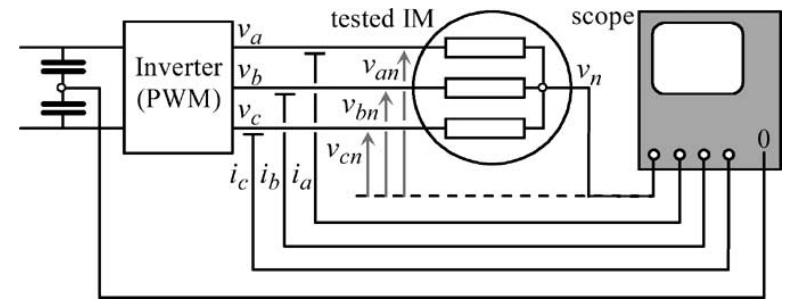

Fig. 2. Signal acquisition for the experimental setup.

memory was used to measure the negative-sequence carrier-signal current and the zero-sequence carrier-signal voltage, (see Fig. 2). For the negative-sequence carrier-signal-current methods the three phase currents were measured and for the zero-sequence-voltage methods the three inverter output phase voltages and the neutral voltage were measured. These signals were then processed offline.

The inverter was operated at a switching frequency of $15 \mathrm{kHz}$, with a dead time of $2 \mu \mathrm{s}$, and a dc-bus voltage of $350 \mathrm{~V}$. A carrier-signal voltage of $\omega_{c}=500 \mathrm{~Hz}$ and $V_{c}=20 \mathrm{~V}$ was used throughout the paper unless stated otherwise.

\section{Methods Based on the Negative-Sequence Carrier-Signal Current}

Methods based on the negative-sequence carrier-signal current usually use filtering to separate the negative-sequence carrier-signal current, which contains the saliency position information in its phase, from the stator current [4]-[9]. This can be simply done by transforming the stator current to a negative-sequence carrier-signal-current reference frame and low-pass filtering. The output has the form of (22)

$$
i_{q d s \_c n}^{c n}=-\mathrm{j} I_{c n} \mathrm{e}^{\mathrm{j} \pm h \theta_{e}} .
$$

The spatial position of the saliency can then be obtained using either a tracking observer, which are zero lag filters, [4], [5], [9] or an arctangent function with lagging filters [6], [7].

Fig. 3 shows the negative-sequence carrier-signal current and the corresponding frequency spectrum for motor \#1 in Table I, operated at rated flux, rated load. From the figure it can be seen that the machine exhibits a rotor-stator slotting saliency, with the harmonic order $h$ being equal to the number of rotor slots per pole pair, in addition to fundamental-frequency-dependent components. Most of the fundamental-frequency-dependent components, and specifically the one at $2 \omega e$, are believed to be caused by saturation [5]-[9]. Nonideal behavior of the inverter has also been reported to cause similar components in the negative-sequence carrier-signal current [7]. 

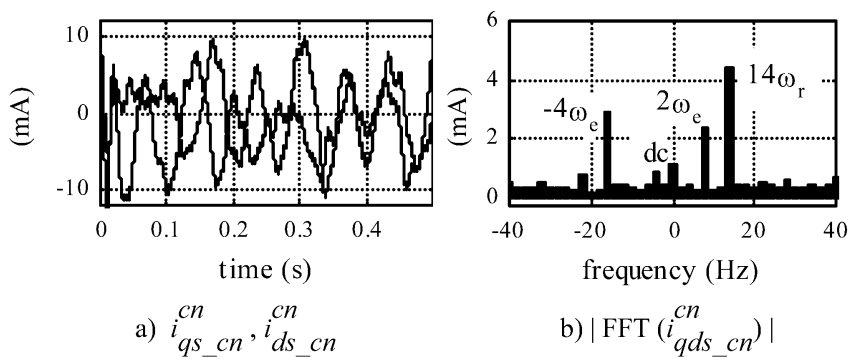

a) $i_{q s \_c n}^{c n}, i_{d s \_c n}^{c n}$

b) $\left|\operatorname{FFT}\left(i_{q d s \_c n}^{c n}\right)\right|$

Fig. 3. (a) Experimentally measured real and imaginary parts of the negative-sequence carrier-signal current. (b) Corresponding frequency spectrum for motor \#1 in Table I. The motor was operated at rated flux, rated load, $\omega_{r}=1 \mathrm{~Hz}, \omega_{e}=4 \mathrm{~Hz}$.
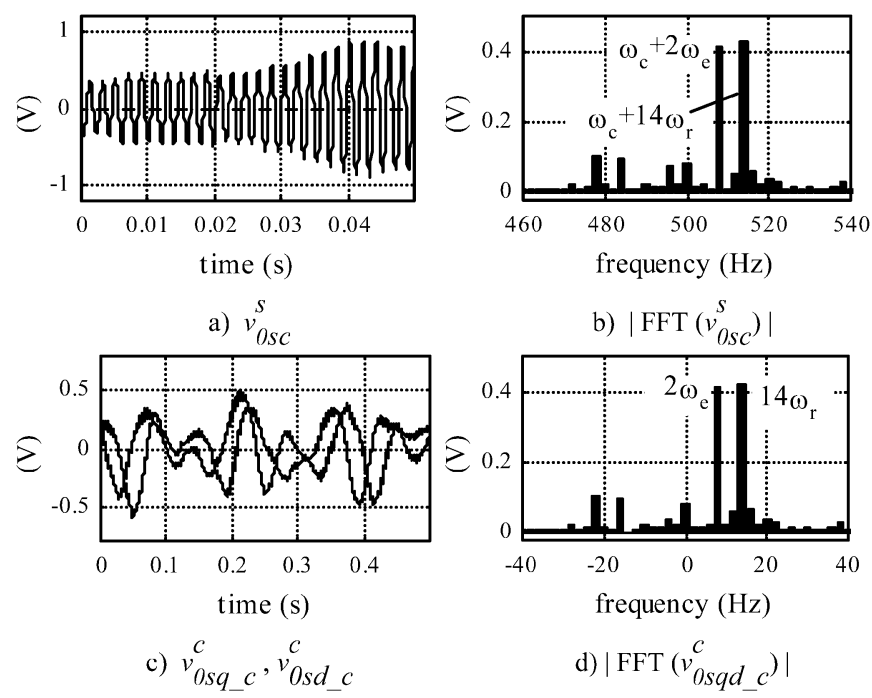

Fig. 4. (a) Experimentally measured zero-sequence carrier-signal voltage. (b) Corresponding frequency spectrum. (c) Real and imaginary parts of the zero-sequence carrier voltage vector. (d) Corresponding frequency spectrum. Motor \#1 was used, operated at rated flux, rated load, $\omega_{r}=1 \mathrm{~Hz}, \omega_{e}=4 \mathrm{~Hz}$.

\section{Methods Based on the Zero-Sequence Carrier-Signal Voltage}

Fig. 4(a) shows the zero-sequence carrier-signal voltage of motor \#1 operated at rated flux, rated load. The motor was star-connected, with the zero-sequence voltage obtained from the measured phase and neutral voltages using (6) (see Fig. 2). The zero-sequence carrier-signal voltage was then isolated using fast-Fourier-transform (FFT)-based signal processing. Alternatively to (6), the neutral voltage can also be measured (23) provided that the inverter does not inject zero-sequence components at frequencies near the carrier frequency

$$
v_{0 s c}^{s}=-v_{n c} .
$$

Fig. 4(b) shows the frequency spectrum of the zero-sequence carrier-signal voltage. From that figure it can be seen that the major fundamental-frequency-dependent component of the spectrum is located at $\omega_{c}+2 \omega_{e}$, which corresponds to a saturation-induced saliency, with a harmonic order $h=2$. It is also observed that, analogous to the negative-sequence carrier-signal current, the zero-sequence carrier-signal voltage also shows content from a rotor-stator slotting saliency located at $\omega_{c}+14 \omega_{r}$, i.e., with the harmonic order $h=14$ equal to the number of rotor bars per pole.
In [13], a time-based measurement technique was used to extract the saliency position information from the zero-sequence carrier-signal voltage. A different approach, similar to the one proposed in [14], is used in this paper, the goal being to allow the use of filtering techniques developed for the negative-sequence carrier-signal current. The zero-sequence carrier-signal voltage in (17) (and similarly for (19)) can be converted to a carrier-synchronous zero-sequence voltage vector using (24). Then, by applying similar synchronous reference frame filtering as used for the negative-sequence carrier-signal current, a single complex vector with the form (25) (multiplied by 2 for convenience) can be isolated from (24) with its phase a function of the saliency position

$$
\begin{aligned}
& v_{0 s q d_{-} c}^{c^{\prime}}=v_{0 s c}^{s} \mathrm{e}^{\mathrm{j} \omega_{c} t}=-\mathrm{j} \frac{V_{0 c h}}{2}\left(\mathrm{e}^{\mathrm{j} h \theta_{e}}-\mathrm{e}^{-\mathrm{j}\left(h \theta_{e}+2 \omega_{c} t\right)}\right) \\
& v_{0 s q d_{-} c}^{c}=-\mathrm{j} V_{0 c h} \mathrm{e}^{\mathrm{j} h \theta_{e}} .
\end{aligned}
$$

This can then be further processed to obtain the saliency position information using the same methods used for the negative-sequence carrier-signal current (22) [4]-[9].

Fig. 4(c) and (d) shows the resulting zero-sequence carrier-signal voltage components and the corresponding frequency spectrum of the zero-sequence carrier-signal voltage shown in Fig. 4(a). It is noted that while the frequency spectrum in Fig. 4(b) corresponds to a scalar signal, the one in Fig. 4(d) corresponds to a complex voltage vector.

\section{Comparison of Methods Based on the Negative-SEQuence CARRIER-Signal CURRENT AND ZERO-SEQUENCE CARRIER-SIGNAL VOLTAGE}

While the results in Figs. 3 and 4 suggest that the negativesequence carrier-signal current and the zero-sequence carriersignal voltage contain similar information, some noticeable differences still exist. Comparison of both signals is presented in this section.

\section{A. Rotor-Position-Dependent Saliencies}

Rotor position estimation using high-frequency signal excitation requires a rotor-position-dependent saliency that couples with the stator windings to produce a measurable signal. Although rotor-position-dependent saliencies are sometimes present in standard machine designs due to the combined effect of stator and rotor slotting [5], [7], [9], [16], they can also be specifically created in several ways [4]-[6]. Using rotor-stator slotting saliencies for rotor position estimation has the advantage of allowing machines with open or semi-closed rotor slots to be suitable for sensorless operation without the need for any further modification or special design. However, not all combinations of rotor-stator slots in open or semi-closed rotor slot machines couple with the stator windings to produce measurable effects.

It was demonstrated in [5] and [9] that, independently of how a saliency is created, in order for it to couple with the stator windings and produce a negative-sequence carrier-signal current (26) needs to be satisfied, assuming the machine has an integer number of slots per pole per phase, where $p$ is the number 

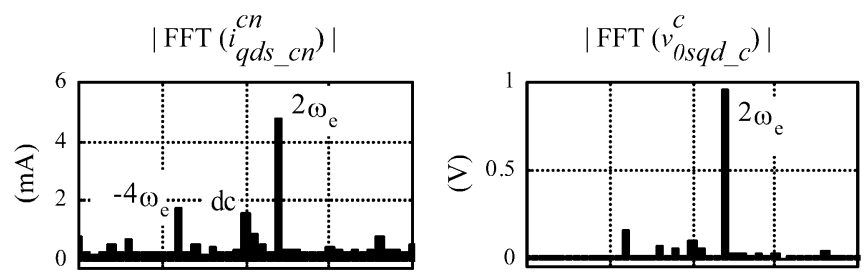

a) Motor \#2 in Table 1 (semi-open rotor slots)
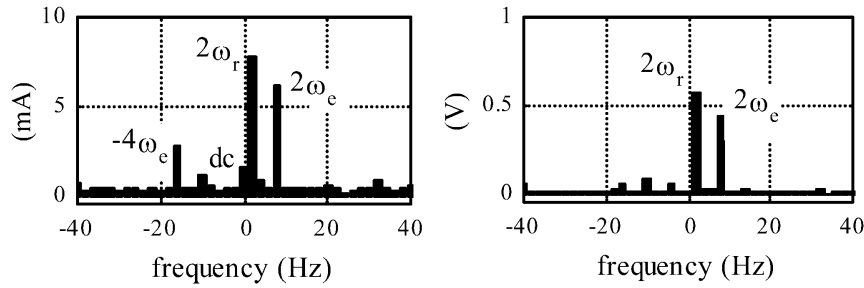

b) Motor \#4 in Table 1 with rotor \#3 (engineered saliency with a period equal to the pole pitch).

Fig. 5. Experimentally measured frequency spectrum of the negative-sequence carrier-signal current (left) and of the zero-sequence carrier voltage complex vector (right) for two different motors. Both motors were operated at rated rated flux, rated load.

of poles and $h_{s p}$ is the saliency harmonic order relative to 360 mechanical degrees

$$
h_{s p}=n \cdot p, \quad n=1,2,4,5,7,8,10, \ldots
$$

Saliencies caused by the combination of rotor and stator slotting produce a permeance waveform that has a fundamental spatial harmonic given by (27), with $S$ and $R$ being equal to the number of stator and rotor slots, respectively. The fundamental of this permeance waveform rotates at the mechanical speed shown in (28) [5], [9]

$$
\begin{aligned}
h_{s p} & =|R-S| \\
\omega_{p} & =\frac{\mathrm{R}}{(\mathrm{R}-\mathrm{S})} \omega_{r m} .
\end{aligned}
$$

The previous analysis, though originally developed for the negative-sequence carrier current, has been found to be valid for the case of zero-sequence carrier-signal voltage. Fig. 5(a) and (b) shows the negative-sequence carrier-signal current frequency spectrum and the zero-sequence carrier-signal voltage vector frequency spectrum for the cases of motor \#2 and motor \#4 using engineered rotor \#3 in Table I. Comparing Figs. 3(b) and 5(b) with Fig. 5(a), it can be seen that in contrast to motors $\# 1$ and \#4, motor \#2 does not show a rotor slotting saliency either in the negative-sequence carrier-signal current or the zerosequence carrier voltage, as predicted by (26) and (27). It can also be seen in Fig. 5(b) that both the negative-sequence carrier-signal current and the zero-sequence carrier-signal voltage have components at $2 \omega_{r}$, which are caused by the engineered saliency.

\section{B. Saturation-Induced Saliencies}

Saturation-induced saliencies, i.e., asymmetries caused by the saturation of the flux paths, have been observed to exist whenever fundamental excitation is present. These saliencies produce components in both the negative-sequence carrier-signal current as well as in the zero-sequence carrier-signal voltage. While saturation-induced harmonics, especially the
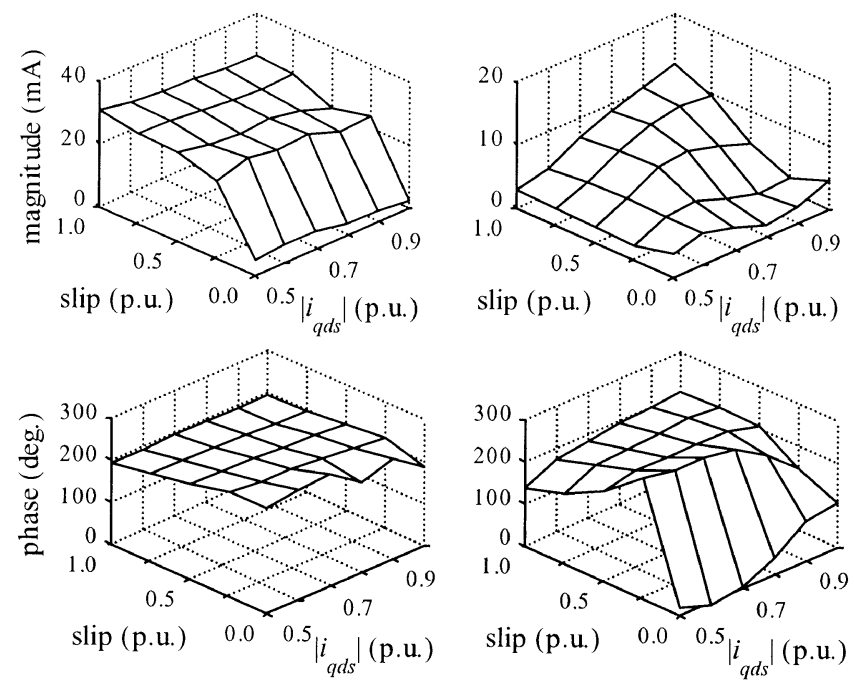

a) Rotor \#1 (closed rotor slots)

b) Rotor \#2 (semi-closed rotor slots)

Fig. 6. Experimentally measured magnitude and phase (relative to the fundamental current) of the $2 \omega_{e}$ harmonic of the negative-sequence carrier-signal current, as a function of the slip frequency and the fundamental current level, for motor \#4 in Table I with (a) semi-closed rotor slots and (b) closed rotor slots. A constant fundamental excitation frequency $\omega_{e}=4 \mathrm{~Hz}$ was used, with the rotor speed varied according to the slip shown in the figure.

one at $2 \omega_{e}$, can be useful for flux angle estimation, they are a disturbance when rotor position estimation is the goal. Unless properly decoupled, they can result in noticeable estimation errors or even stability problems.

Negative-Sequence Carrier-Signal Current: Saturation-induced saliencies and their effects on the negative-sequence carrier-signal current are strongly influenced by the machine design, with one of the most relevant aspects being the rotor slot design [5], [8]. Fig. 6(a) and (b) shows the magnitude and phase angle of the $2 \omega_{e}$ saturation-induced harmonic of the negative-sequence carrier-signal current, as a function of the slip frequency and the fundamental current level, for the cases of motor \#4 in Table I with semi-closed rotor slots and closed rotor slots, respectively. From the figure it can be seen that the phase of the $2 \omega_{e}$ harmonic of the negative-sequence carrier current signal for the case of closed rotor slots, relative to the stator current angle, exhibits only relatively minor variations with slip, suggesting that it is mostly influenced by the stator leakage flux [8]. Larger variations are observed for the case of semi-closed slots, especially for low slips, suggesting that other fluxes in the machine play an important role for closed rotor slot machines. In addition to the rotor slot design, a large set of other design characteristics seem to influence the behavior of saturation-induced saliencies, as demonstrated in [8] where noticeable differences were observed for machines with relatively similar designs and sizes.

Zero-Sequence Carrier-Signal Voltage: The study of saturation-induced saliencies for zero-sequence carrier-signal-voltage-based methods has not received as much attention as the negative-sequence carrier-signal-current-based methods [13], [15].

Fig. 7(a) shows the magnitude and phase (using the fundamental current angle as the reference angle) of the $2 \omega_{e}$ frequency component of the zero-sequence carrier-signal 


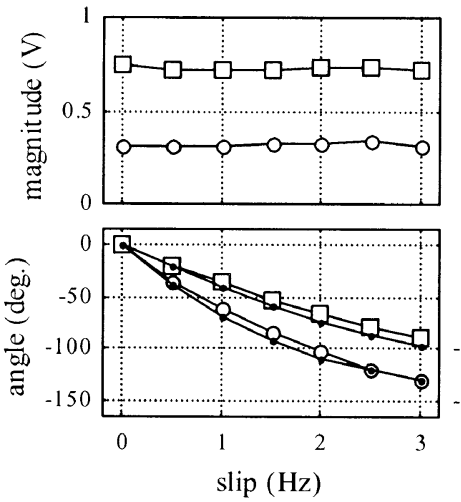

a) Semi-open rotor slots
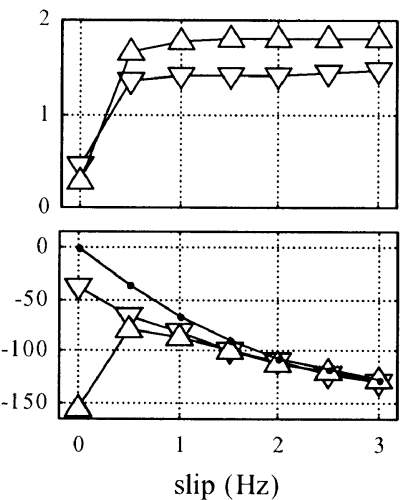

b) Closed-rotor slots
Fig. 7. Experimentally measured magnitude and phase (relative to the stator current vector), of the $2 \omega_{e}$ component of the zero-sequence carrier-signal voltage, for the following motors in Table I. (a) Motor \#1 ( $\bigcirc$ ) and \#2 ( $\square$ ) with semi-closed rotor slots. (b) Motor \#3 $(\triangle)$ and \#4 $(\nabla)$ with closed rotor slots. The estimated angle $2 \theta_{r f}$ for motors \#1 and \#2 in the left column, and for motor \#3 in the right column, are marked with $(\bullet)$. The motors were operated at rated flux, the load being varied according to the slip.

voltage (8) as a function of the slip, for two different motors with semi-open rotor slots, while Fig. 7(b) shows it for two motors with closed rotor slots. The machines were current regulated, operating under indirect field orientation at rated flux (see Fig. 1).

To better understand the behavior of the angles seen in Fig. 7, the theoretical angle of the rotor flux, $\theta_{r f}$, with respect to the stator current vector was obtained. If the saturation giving rise to the $2 \omega_{e}$ component of the zero-sequence carrier-signal voltage were caused by the rotor flux, it would have an angle $2 \theta_{r f}$. This angle is also shown in Fig. 7. Using the rotor flux angle as a comparison for the experimental results was a matter of convenience due to the simplicity of its estimation, as it depends only on the slip and the rotor time constant.

Two interesting facts can be observed from Fig. 7. First, a very good agreement can be seen between the measured and estimated angles for the two different machines with semi-open rotor slots [Fig. 7(a)]. This strongly supports the theory that the $2 \omega_{e}$ component of the zero-sequence carrier-signal voltage is caused by the rotor flux. Second, significant differences are observed between the estimated and measured angles for no-load operation of closed-rotor-slot machines. It is also seen that while the magnitude of the $2 \omega_{e}$ component of the negative-sequence carrier-signal voltage remains relatively constant for the case of open rotor slot machines, it noticeably decreases at no load for closed-rotor-slot machines.

A potential explanation for the behavior observed in closedrotor-slot machines is the following. Fig. 8(a) schematically shows the currents and rotor flux position for a two-pole machine operated at rated flux, no-load condition. Under these conditions, all of the fluxes in the machine have the same phase angle but travel through different portions of the iron. The rotor flux would be expected to cause saturation of the stator teeth and rotor teeth, as marked in the figure. However, because of the narrow rotor slot bridges, stator leakage flux crossing the air gap and returning along the top of rotor (i.e., a form of zigzag flux) could cause saturation of the rotor slot bridges. If that occurs,

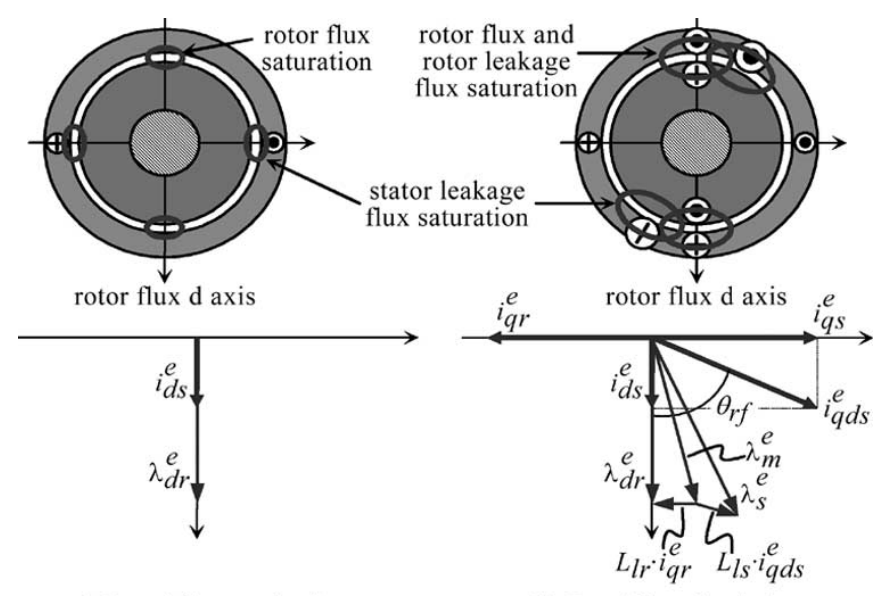

a) Rated flux, no load

b) Rated flux, loaded

Fig. 8. Schematic representation of the relative position between the fluxes and the stator current for the case of rated-flux, no-load, and rated-flux-loaded two-pole machine, shown in a rotor flux reference frame.

two different regions of the machine, separated $90^{\circ}$ from each other, would be saturated, as seen if Fig. 8(a). This sort of saturation would appear from the stator winding to be a relatively homogenous saturation of the machine and, as a consequence, the magnitude of the $2 \omega_{e}$ component of the zero-sequence carrier-signal voltage would be small in magnitude. In addition, the phase of the $2 \omega_{e}$ component would depend on the combined effect of the two phenomena described above and would not, therefore, be aligned with any specific flux in the machine. As the load increases, the rotor leakage flux will strongly saturate the rotor slot bridges, spatially coinciding with the rotor flux [Fig. 8(b)]. This additional saturation dominates the saturation-induced saliency and, therefore, the $2 \omega_{e}$ component of the zero-sequence carrier-signal voltage.

On the other hand, for the case of semi-open or open-rotor-slot machines, saturation of the stator/rotor teeth caused by the rotor flux is the dominant effect under no-load operation. This effect is only boosted by the rotor leakage flux when the machine is loaded, with the phase angle of the $2 \omega_{e}$ component of the zero-sequence carrier-signal voltage almost coinciding with the rotor flux.

\section{PWM-Switching-Excitation ZeRo-Sequence-Voltage Measurement-Based SENSORLesS Methods}

Switching harmonics, due to the PWM operation of the inverter, have also been shown to be useful for saliency position estimation [3], [15]-[18]. In the technique proposed in [16], the instantaneous line-to-neutral voltage (29) (see Fig. 2) is measured after applying two adjacent switching states and forcing the commutation of switches in the third phase by substituting the zero state with two active states for a short period of time. A complex voltage vector $v_{q d \sigma}(30)$ is then obtained from the three measurements

$$
\begin{aligned}
v_{\sigma} & =\frac{1}{3}\left(v_{a n}+v_{b n}+v_{c n}\right) \\
v_{q d \sigma} & =\frac{2}{3}\left(v_{\sigma a}+v_{\sigma b} \mathrm{e}^{\mathrm{j} 2 \pi / 3}+v_{\sigma c} \mathrm{e}^{\mathrm{j} 4 \pi / 3}\right) .
\end{aligned}
$$



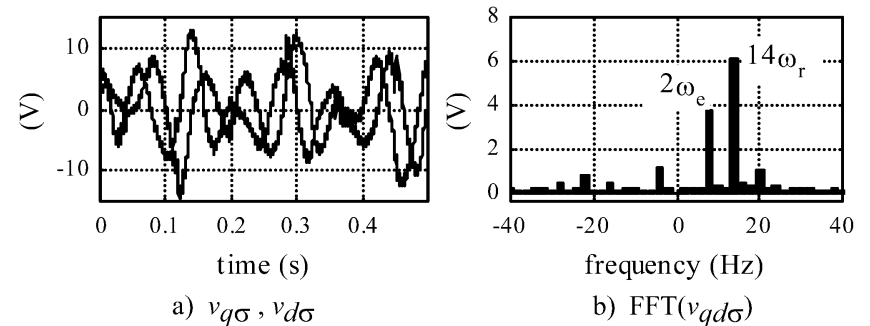

Fig. 9. (a) Experimentally measured real and imaginary parts of the complex vector $v_{q d \sigma}$. (b) Corresponding frequency spectrum for motor \#1 in Table I. The motor was operated at rated flux, rated load.

As this technique relies on the measurement of the zero-sequence voltage, similar to the carrier-signal method described above, they are expected to behave similarly. Fig. 9 shows the complex voltage vector defined by (30) and its frequency spectrum for the case of motor \#1 in Table I. The results shown are very similar to those presented earlier in Fig. 4 for the zero-sequence carrier-signal voltage vector.

\section{COMPARISON OF PWM-SWITCHING-BASED AND Carrier-Voltage-Based Zero-Sequence-Voltage METHODS}

Although both zero-sequence-voltage-based methods are expected to exhibit similar behaviors, some major differences exist in their practical implementation. Some similarities and differences between both methods are analyzed in this section.

\section{A. Rotor-Position-Dependent Saliencies}

Results reported in [3] and [16], as well as those shown in Fig. 9, were obtained using machines with open rotor slots. It was previously shown in Section III-A that both the negative-sequence carrier-signal current and the zero-sequence carrier-signal voltage had similar restrictions for the coupling of a rotor-stator saliency with the stator windings.

In [16], it was concluded that in order for rotor slotting saliencies to create a measurable complex voltage vector (30) the number of rotor bars per pole pair could not be a multiple of three, with the number of stator slots not having any influence. This criteria differs from the one established by (26)-(28), and can be shown to be a particular case. Fig. 10 shows the complex voltage vector defined by (30) and its frequency spectrum for the case of motor \#2 in Table I. It is noted that motor \#2 is from the same manufacturer and with a similar design as motor \#1. From Fig. 10 it is observed that the voltage vector does not show a component due to the rotor slotting saliency. While this result does not follow the rule stated in [16], it is consistent with the results predicted by (26)-(28).

\section{B. Saturation-Induced Saliencies and Flux Angle Estimation}

As was the case for the negative-sequence carrier-signal current and zero-sequence carrier-signal voltage, saturation-induced saliencies also influence the complex voltage vector (30) [3], [16]. While saturation-induced harmonics can potentially be useful for flux angle estimation, they are a disturbance when rotor position estimation is the goal. Their compensation

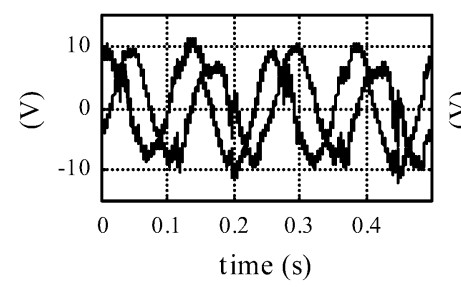

a) $v_{q \sigma}, v_{d \sigma}$

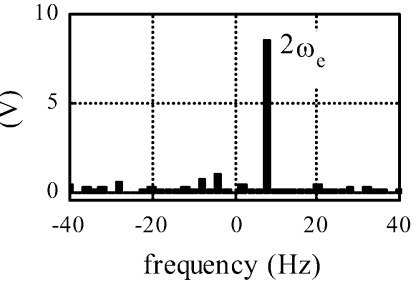

b) $\operatorname{FFT}\left(v_{q d \sigma}\right)$
Fig. 10. (a) Experimentally measured real and imaginary parts of the complex vector $v_{q d \sigma}$. (b) Corresponding frequency spectrum for motor \#2 in Table I. The motor was operated at rated flux, rated load.

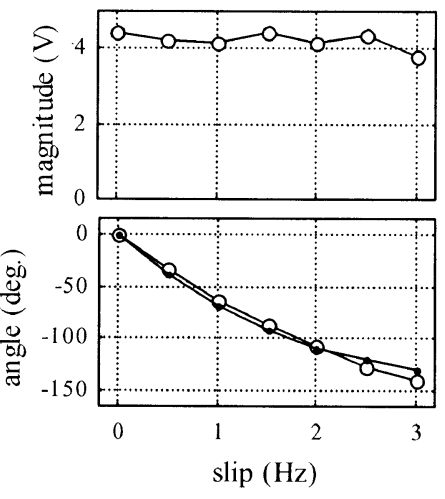

a) Motor \#1
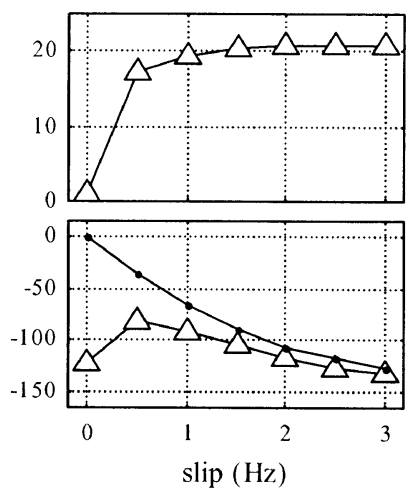

b) Motor \#3, closed-rotor slots
Fig. 11. Experimentally measured magnitude and phase, relative to the stator current vector, of the $2 \omega_{e}$ component of the complex voltage vector $v_{q d \sigma}$ for (a) semi-closed rotor slots and (b) closed rotor slots. The motors were operated at rated flux, the load being varied according to the slip.

to obtain reliable and accurate rotor position estimation was demonstrated in [16].

Fig. 11 shows the magnitude and phase of the $2 \omega_{e}$ frequency component of the complex voltage vector $v_{q d \sigma}$ for the two different machines, with open and semi-closed rotor slots. Comparing Figs. 11 and 7, the similarities between the behavior of saturation-induced saliencies for the case of the zero-sequence PWM switching voltage vector and the zero-sequence carriersignal voltage vector are evident. This strongly supports the idea that both techniques share the same physical principles regardless of the differences in their implementation.

It is finally noted that the similarities between the results shown in Figs. 7 and 11 suggest that the commissioning process for the compensation of saturation-induced saliencies described in [16] would be valid for the case of zero-sequence carriersignal voltage methods.

\section{Influence of the Carrier Frequency}

All the results using the zero-sequence carrier voltage presented so far used a carrier frequency of $\omega_{c}=500 \mathrm{~Hz}$. Experiments using different carrier frequencies were also performed. Fig. 12 shows the magnitudes of the rotor-stator slotting saliency and of the $2 \omega_{e}$ saturation-induced component for various carrier frequencies. From Fig. 12 it is observed that the magnitude of the resulting rotor-position-dependent component of the zero-sequence carrier voltage is almost constant, independent of the carrier frequency. In addition, the saturation-induced component of the zero-sequence carrier-signal 


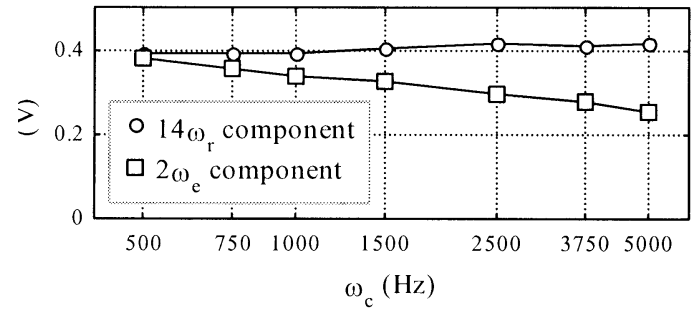

Fig. 12. Experimentally measured magnitude of the $2 \omega_{e}$ and $14 \omega_{r}$ components of the zero-sequence carrier-signal voltage as a function of the carrier frequency for motor \#1 in Table I. The motor was operated at rated flux, rated load. A carrier voltage of peak value of $20 \mathrm{~V}$ was used.

voltage is seen to decrease as $\omega_{c}$ increases. This suggests that higher values of $\omega_{c}$ would improve the robustness for decoupling saturation-induced saliencies since their magnitude relative to the rotor position dependent component is decreased. It is also interesting to notice that for the case of $\omega_{c}=5000 \mathrm{~Hz}$ (one-third of the switching frequency), the ratio between both components of the zero-sequence carrier voltage is similar to the observed for the case of the complex voltage vector $v_{q d \sigma}$ in Fig. 9. This would be expected, since as $\omega_{c}$ increases the results should be closer to the quasiinstantaneous response measured by the complex voltage vector $v_{q d \sigma}$.

\section{Test Voltage Injection}

One of the advantages of PWM-switching-based methods with respect to carrier-signal-injection-based methods, according to [16], was that no high-frequency carrier-signal voltage has to be injected. However, this advantage does not come without some penalties. To obtain the complex voltage vector (30), the regular switching pattern has to be modified, a zero-voltage-switching state vector being replaced for a short period of time by two opposite active switching state vectors. The modification of the regular switching pattern forces all six power devices of the inverter to switch simultaneously, increasing inverter losses.

A second advantage cited for PWM-switching-based methods with respect to carrier-signal-injection-based methods is the reduction of the resulting high-frequency currents and, as a consequence, the associated torque ripple. However, this effect can be mitigated for the case of carrier-signal-injection-based methods by increasing the carrier frequency. As the carrier frequency increases (keeping the carrier voltage magnitude $V_{c}$ constant), the carrier-signal current and, consequently, the associated torque ripple, will decrease. In addition, as seen in Fig. 12, increasing the carrier frequency was found to increase the ratio of the rotor-position-dependent saliency with respect to the saturation-induced saliencies.

\section{CONCLUSION}

Three different saliency-based sensorless methods for ac machines have been analyzed in this paper: negative-sequence carrier-signal-current-based methods, zero-sequence carrier-signal-voltage-based methods, and zero-sequence PWM-switching-voltage-based methods. From the analysis, the following conclusions are reached.
- All the methods can be used for rotor position estimation and field orientation, since rotor-position-dependent saliencies, as well as saturation-induced saliencies, produce measurable effects.

- The same restrictions have been observed for all of the methods that result in rotor-position-dependent saliencies, and specifically rotor-stator slotting saliencies, coupling with the stator windings and producing measurable effects.

- Saturation-induced saliencies in the zero-sequence-voltage methods, independent of whether they are carrier-signal based or PWM-switching based, have been shown to depend on the main flux for the case of open and semi-closed rotor slot machines. This suggests that they could be useful for flux angle estimation. Flux angle estimation seems much more difficult for the case of closed-rotor-slot machines, as the experiments presented in this paper show that saturation-induced saliencies for those machines are influenced by leakage fluxes.

- Zero-sequence-voltage carrier-based methods and PWM-switching zero-sequence-voltage-based methods have been shown to share the same physical principles and to provide similar results. The differences between them would be mostly related to implementation aspects and it is not obvious that either method can be viewed as superior.

- The nonideal behavior of the inverter, as well as other secondary effects like high-frequency phenomena caused by long cables lengths, shielding, and grounding practices, has been observed to influence the methods in different ways. These effects might give rise to relevant differences in the implementation, robustness, and accuracy of the various methods studied. However, research on these issues is still ongoing and definite conclusions cannot be drawn at this time.

- There are a number of relevant machine-oriented issues that are the focus of ongoing research, such as the effect of motor design on attainable accuracy and resolution, and the number of harmonics needed to achieve a specified accuracy. These issues also affect the simplicity/complexity of the relative implementation. The ongoing research in this area is expected to produce further improvements in the relative robustness, performance, and cost effectiveness of the methods and may lead to design evolution of existing machines to make them more competitive when using such methods.

\section{ACKNOWLEDGMENT}

The authors wish to acknowledge the support and motivation provided by the University of Oviedo, Gijón, Spain, Ford Motor Company, and the Wisconsin Electric Machines and Power Electronics Consortium (WEMPEC) of the University of Wisconsin, Madison.

\section{REFERENCES}

[1] H. Tajima and Y. Hori, "Speed sensorless field orientation control of the induction machine," IEEE Trans. Ind. Applicat., vol. 29, pp. 175-180, Jan./Feb. 1993. 
[2] H. Kubota and K. Matsuse, "The improvement of performance at low speed by offset compensation of stator voltage in sensorless vector controlled induction machines," in Conf. Rec. IEEE-IAS Annu. Meeting, San Diego, CA, Oct. 1996, pp. 257-261.

[3] J. Holtz, "Sensorless control of induction motor drives," Proc. IEEE, vol. 90, pp. 1359-1394, Aug. 2002

[4] P. L. Jansen and R. D. Lorenz, "Transducerless position and velocity estimation in induction and salient AC machines," IEEE Trans. Ind. Applicat., vol. 31, pp. 240-247, Mar./Apr. 1995.

[5] M. W. Degner, "Flux, position and velocity estimation in AC machines using carrier frequency signal injection," Ph.D. dissertation, Dept. Elect. Comput. Eng., Univ. Wisconsin, Madison, WI, 1998.

[6] J. Cilia, G. M. Asher, and K. G. Bradley, "Sensorless position detection for vector controlled induction motor drives using an asymmetric outersection cage," in Conf. Rec. IEEE-IAS Annu. Meeting, San Diego, CA, Oct. 1996, pp. 286-292.

[7] N. Teske, G. M. Asher, M. Sumner, and K. J. Bradley, "Encoderless position estimation for symmetric cage induction machines under loaded conditions," IEEE Trans. Ind. Applicat., vol. 37, pp. 1793-1800, Nov./Dec. 2001

[8] F. Briz, M. W. Degner, A. Diez, and R. D. Lorenz, "Static and dynamic behavior of saturation-induced saliencies and their effect on carrier signal based sensorless AC drives," IEEE Trans. Ind. Applicat., vol. 38, pp. 670-678, May/June 2002.

[9] M. W. Degner and R. D. Lorenz, "Position estimation in induction machines utilizing rotor bar slot harmonics and carrier frequency signal injection," IEEE Trans. Ind. Applicat., vol. 36, pp. 736-742, May/June 2000.

[10] L. A. S. Ribeiro, M. W. Degner, F. Briz, and R. D. Lorenz, "Comparing carrier frequency voltage and current injection for the estimation of flux, position and velocity in sensorless AC drives," in Conf. Rec. IEEE-IAS Annu. Meeting, St. Louis, MO, Oct. 1998, pp. 452-459.

[11] J. Ha and S. Sul, "Sensorless field-orientation control of an induction machine by high-frequency signal injection," IEEE Trans. Ind. Applicat., vol. 35, pp. 45-51, Jan./Feb. 1999.

[12] M. J. Corley and R. D. Lorenz, "Rotor position and velocity estimation for a salient-pole permanent magnet synchronous machine at standstill and high speeds," IEEE Trans. Ind. Applicat., vol. 34, pp. 784-789, July/Aug. 1998.

[13] A. Consoli, G. Scarcella, and A. Testa, "A new zero-frequency flux-position detection approach for direct-field-oriented-control drives," IEEE Trans. Ind. Applicat., vol. 36, pp. 797-804, May/June 2000.

[14] P. L. Jansen, W. J. Premerlani, and L. J. Garcés, "System and method for sensorless rotor tracking of induction machines," U.S. Patent 6388420 B1, May 2002.

[15] Giebeler and Christoph, "Implementation and evaluation of amplitudebased self-sensing torque control for induction machines," M.S. thesis, Dept. Elect. Comput/ Eng., Univ. Wisconsin, Madison, WI, 2002.

[16] J. Holtz and H. Pan, "Elimination of saturation effects in sensorless position controlled induction motors," in Conf. Rec. IEEE-IAS Annu. Meeting, Pittsburgh, PA, Oct. 2002, CD-ROM.

[17] M. Schroedl, "Sensorless control of AC machines at low speed and standstill based on the inform method," in Conf. Rec. IEEE-IAS Annu. Meeting, Chicago, IL, Sept.-Oct. 2001, CD-ROM.

[18] T. M. Wolbank and J. Machl, "A modified PWM scheme in order to obtain spatial information of AC machines without mechanical sensors," in Proc. IEEE APEC'02, Dallas, TX, Mar. 2002, CD-ROM.

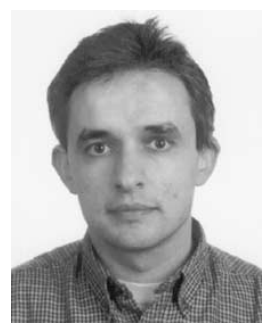

Fernando Briz (A'96-M'99) received the M.S. an $\mathrm{Ph} . \mathrm{D}$. degrees from the University of Oviedo, Gijón, Spain, in 1990 and 1996, respectively.

From June 1996 to March 1997, he was a Visiting Researcher at the University of Wisconsin, Madison. $\mathrm{He}$ is currently an Associate Professor in the Electrical Engineering Department, University of Oviedo. His topics of interest include control systems, high-performance ac drives control, sensorless control, and digital signal processing.

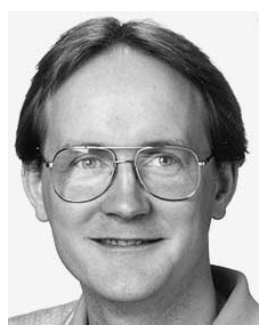

Michael W. Degner (S'95-A'98-M'99) received the B.S., M.S., and Ph.D. degrees in mechanical engineering from the University of Wisconsin, Madison, in 1991, 1993, and 1998, respectively, with a focus on electric machines, power electronics, and control systems.

In 1998, he joined the Ford Research Laboratory of Ford Motor Company, Dearborn, MI, where his research focused on the use of power electronics in automotive applications. He is currently Project Leader of the Power Electronics and Electric Drives group in the Sustainable Mobility Technologies Laboratory of Ford Research and Advanced Engineering. His interests include control systems, electric machines and drives, power electronics, and mechatronics.

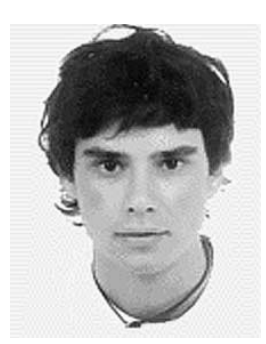

Pablo García (S'02) was born in Spain in 1975. He received the M.E. degree in industrial engineering in 2001 from the University of Oviedo, Gijón, Spain, where he is currently working toward the Ph.D. degree in electrical engineering.

His research interests include sensorless control and diagnosis of induction motors, neural networks, and digital signal processing.

Mr. García was awarded a fellowship of the Personnel Research Training Programme funded by the Spanish Ministry of Science and Technology in 2001

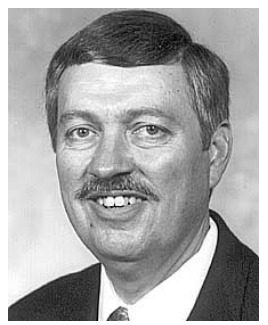

Robert D. Lorenz (S'83-M'84-SM'91-F'98) received the B.S., M.S., and Ph.D. degrees from the University of Wisconsin, Madison, and the M.B.A. degree from the University of Rochester, Rochester, NY.

Since 1984, he has been a member of the faculty of the University of Wisconsin, Madison, where he is the Mead Witter Foundation Consolidated Papers Professor of Controls Engineering in both the Department of Mechanical Engineering and the Department of Electrical and Computer Engineering. He is Co-Director of the Wisconsin Electric Machines and Power Electronics Consortium, which celebrated its 20th anniversary in 2001. It is the largest industrial research consortium on motor drives in the world. He is also the thrust leader for control and sensor integration in the Center for Power Electronic Systems, an NSF Engineering Research Center (ERC) which is a joint ERC with Virgina Polytechnic Institute and State University, Rensselaer Polytechnic Institute, University of Puerto Rico-Mayaguez, and North Carolina A\&T. From 1972 to 1982, he was a member of the research staff at the Gleason Works, Rochester, NY, working principally on high-performance drives and synchronized motion control. He was a Visiting Research Professor in the Electrical Drives Group, Catholic University of Leuven, Leuven, Belgium, in the summer of 1989 and in the Power Electronics and Electrical Drives Institute, Technical University of Aachen, Aachen, Germany, in the summers of 1987, 1991, 1995, 1997, and 1999, where he also was the SEW Eurodrive Guest Professor from September 1, 2000 until July 7, 2001. In 1969-1970, he conducted Master thesis research in adaptive control of machine tools at the Technical University of Aachen. His current research interests include sensorless electromagnetic motor/actuator technologies, real-time signal processing and estimation techniques, precision multiaxis motion control, and ac/dc drive and high-precision machine control technologies. He has authored more than 160 published technical papers and is the holder of 16 patents, with two more pending.

Dr. Lorenz was the IEEE Industry Applications Society (IAS) President for 2001, a Distinguished Lecturer of the IAS for 2000/2001, immediate past Chair of the IAS Awards Department, and past Chairman of the IAS Industrial Drives Committee, and is a member of the IAS Industrial Drives, Electric Machines, Industrial Power Converter, and Industrial Automation and Control Committees. $\mathrm{He}$ is also the current Chair of the Periodicals Committee for the IEEE Technical Activities Board. He is a member of the IEEE Sensor Council AdCom. He was awarded the 2003 IEEE IAS Outstanding Achievement award, which honors his outstanding contributions and technological developments in the application of electricity to industry. He has won 15 prize paper awards. He is a Member of the American Society of Mechanical Enginees, Instrument Society of America, and The International Society for Optical Engineers. He is a Registered Professional Engineer in the States of New York and Wisconsin. 